\title{
Magneto-Optical and Optical Investigation of the Surface Region of Ion implanted Garnet Films
}

\author{
KALANDADZE LALI*
}

Department of Physics, Batumi Shota Rustaveli State University, Ninoshvili 35, Batumi 6010, Georgia

In the present work we have shown the efficiency of complex investigation of the ion implanted garnet surface region by magneto-optical and optical methods. We have investigated the optical and magneto-optical properties of the ion-implanted $(\mathrm{YBiCaSm})_{3}(\mathrm{FeGeSi})_{5} \mathrm{O}_{12}$ garnet films. It has shown that ion implantation influences significantly the magneto-optical properties of the garnet films and practically does not change its optical characteristics. We have also determined the spectral dependences of the component of the tensor of dielectric permittivity for the surface of ion implanted $(\mathrm{YBiCaSm})_{3}(\mathrm{FeGeSi})_{5} \mathrm{O}_{12}$ ferrite-garnet films before and after implantation process. These calculations let us evaluate the influence of implantation on an electronic energy structure of the surface layer for the sample.

DOI: 10.12693 /APhysPolA.127.582

PACS: $75.70 . \mathrm{Cn}, 78.20 . \mathrm{Ls}$

\section{Introduction}

Nowadays, magneto-optical research methods are widely used for examination of ultrafine magnetic structures with insertions that have the sizes of structural and magnetic heterogeneities less than $1000 \AA$. Magnetooptical investigation of these types of structures such as: magnetic fluids, thin discontinuous metal films, heterogenic glasses and etc. are the subject of overall interest conditioned by both theoretical and practical significance [1]. Besides, the ultrafine magnetic structures with structural heterogeneities, there are the media, heterogeneities of which carry magnetic character.

Heterogeneous magnetic structures could be formed in the surface layer of the solid by the different kind of outer impact, for instance, by the ion implantation. Implantation affects the physical-chemical properties, phase composition and surface structure of the solid. As a result, different radiation defects may cause the heterogeneity of the local magnetic properties.

There are considerable scientific and practical interests regarding investigation of the characteristic properties of magneto-optical behavior of the ion-implanted garnet films [2, 3].

In the present work we give the results of magnetooptical and optical investigations of the properties of the surface regions of the ion-implanted $(\mathrm{YBiCaSm})_{3}(\mathrm{FeGeSi})_{5} \mathrm{O}_{12}$ garnet films.

The magneto-optical method of investigation of the surface of the solid [4], in which use is made of the fact that the light reflected from a magnetized medium penetrates into a sample to a small depth and the magnetooptical reflection effects are proportional to the magnetization of the surface layer, has already been widespread.

This investigative method lets us take over changes in the structure of an elementary cell of the solid, examine

*e-mail: lali62@mail.ru superficial magnetic transformation and discover heterogeneous magnetic structures.

\section{Experimental details}

In our experiments we used $(\mathrm{YBiCaSm})_{3}(\mathrm{FeGeSi})_{5} \mathrm{O}_{12}$ garnet films of $1.3 \mu \mathrm{m}$ thickness prepared by means of liquid phase epitaxy on $\mathrm{Gd}_{3} \mathrm{Ga}_{5} \mathrm{O}_{12}$ substrates with the (111) crystallographic orientation. The thickness of the substrates was $450 \mu \mathrm{m}$.

The implantation process was carried out at the room temperature by $\mathrm{Ne}^{+}$ions with energy of $100 \mathrm{keV}$ and with various doses $(0.5-2.5) \times 10^{14}$ ion $/ \mathrm{cm}^{2}$. During this process, permeation depth of the implanted ions covered $0.1 \mu \mathrm{m}$, maximum quantity of the implanted ions went to the depth $0.07 \mu \mathrm{m}$.

For the magneto-optical investigation of a garnet surface we have chosen the odd-magnetization equatorial Kerr effect (EKE). The EKE consists in a change in the intensity of linearly polarized light reflected from the sample in the case of reversal of magnetization of the sample. It can be written as

$$
\delta=\frac{I_{H}-I_{H=0}}{I_{H=0}},
$$

where $I_{H}$ and $I_{H=0}$ are, respectively, the intensities of light reflected from the magnetized and demagnetized sample.

The tensor of dielectric permittivity for bulk ferromagnetic materials can be represented as

$$
\varepsilon=\left(\begin{array}{ccc}
\varepsilon & \mathrm{i} \varepsilon^{\prime} & 0 \\
-i i \varepsilon^{\prime} & \varepsilon & 0 \\
0 & 0 & \varepsilon_{0}
\end{array}\right),
$$

where $\varepsilon=\varepsilon_{1}-\mathrm{i} \varepsilon_{2} ; \varepsilon_{0}=\varepsilon_{01}-\mathrm{i} \varepsilon_{02}$, and $\varepsilon^{\prime}=\varepsilon_{1}^{\prime}-\mathrm{i} \varepsilon_{2}^{\prime}$.

The EKE is related to the tensor components of the dielectric permittivity as follows:

$$
\delta=\frac{2 \sin 2 \phi\left(A \varepsilon_{1}^{\prime}+B \varepsilon_{2}^{\prime}\right)}{A^{2}+B^{2}},
$$

Where $A=\varepsilon_{2}\left(2 \varepsilon_{1}^{\prime} \cos ^{2} \varphi-1\right), B=\left(\varepsilon_{2}^{2}-\varepsilon_{1}^{2}\right) \cos ^{2} \phi+\varepsilon_{1}$ $-\sin ^{2} \phi$ and $\phi$ is the angle of light incidence on the sample. 
The optical constants were determined using the Avery method [5].

\section{Results and discussion}

The research of the spectral dependences of the EKE for unimplanted and ion-implanted with various dose $(\mathrm{YBiCa})_{3}(\mathrm{FeGe})_{5} \mathrm{O}_{12}$ ferrite-garnet films has shown that the ion implantation significantly influences on the magneto-optical properties of the ferrite-garnet films.

For example, Fig. 1 presents the dependences of the EKE on the quantum energy of incident light $\hbar \omega$ for the $(\mathrm{YBiCaSm})_{3}(\mathrm{FeGeSi})_{5} \mathrm{O}_{12}$ films before and after the implantation process with various doses. The angle of light incidence on the sample $\phi=70^{\circ}$.

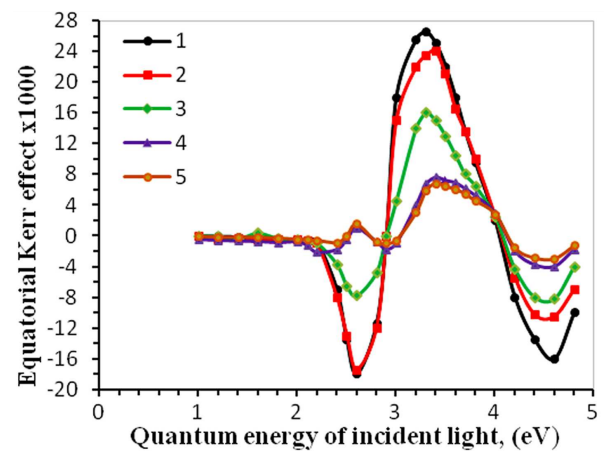

Fig. 1. Dependences of the EKE on the quantum energy of incident light $\hbar \omega\left(\phi=70^{\circ}\right)$ for the $(\mathrm{YBiCaSm})_{3}(\mathrm{FeGeSi})_{5} \mathrm{O}_{12}$ films before (curve 1) and after the process of implantation with various doses: $0.5 \times 10^{14}$ (curve 2$), 1.5 \times 10^{14}$ (curve 3$), 2.0 \times 10^{14}$ (curve 4) and $2.5 \times 10^{14}$ ion $/ \mathrm{cm}^{2}$ (curve 5).

According to Fig. 1 the magneto-optical spectrum of unimplanted garnet surface has the magneto-optical maxima in the region of light quantum energies $\hbar \omega=2.6$, 3.4 , and $4.5 \mathrm{eV}$. Most garnet films are characterized by the similar peaks [6]. For the implantation with the minor doses the spectrum character of the EKE is practically the same. But it significantly changes when doses are $(1.5-2.5) \times 10^{14}$ ion $/ \mathrm{cm}^{2}$. Specifically, an increase of the implantation dose causes a decrease of the magnetooptical maxima in the region of light quantum energies $\hbar \omega=3.4$ and $4.5 \mathrm{eV}$, which may be connected with the split of $\mathrm{FeO}_{4}$ and $\mathrm{FeO}_{6}$ molecular complexes, where intensive electron transition takes place with energy more than $3 \mathrm{eV}$. But the increase of the implantation dose causes reduction of the negative magneto-optical maximum in the region of light quantum energy $\hbar \omega=2.6 \mathrm{eV}$ with the effect which gets in the end a new positive meaning. The similar change of sign of the effect in the region of light quantum energy $\hbar \omega=2.6 \mathrm{eV}$ has been observed for the $(\mathrm{YBiCa})_{3}(\mathrm{FeGe})_{5} \mathrm{O}_{12}$ garnet films after specific implantation dose $\left(1.5-2.5 \times 10^{14}\right.$ ion $/ \mathrm{cm}^{2}$ with increasing external magnetic field. For example, Fig. 3 presents the dependences of the EKE on the quantum energy of incident light $\hbar \omega$ for the films after the process of implantation with doses: $(2.0-2.5) \times 10^{14} \mathrm{ion} / \mathrm{cm}^{2}$ at $H_{\sim}=0.2 \mathrm{kOe}$ and $H_{\sim}=2.5 \mathrm{kOe}$.

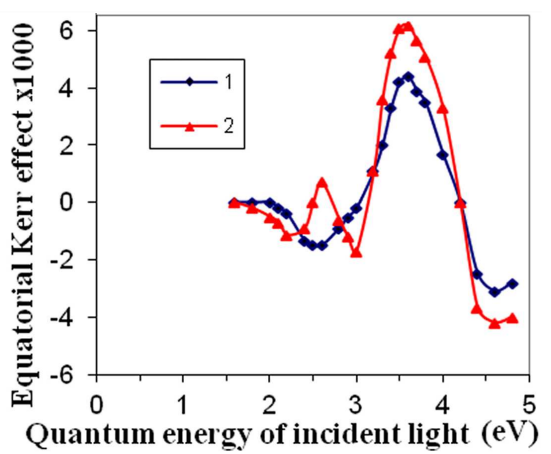

Fig. 2. Dependences of the EKE on the quantum energy of incident light $\hbar \omega\left(\phi=70^{\circ}\right)$ for the $(\mathrm{YBiCaSm})_{3}(\mathrm{FeGeSi})_{5} \mathrm{O}_{12}$ garnet film after the process of implantation with dose: $2.0 \times 10^{14}$ ion $/ \mathrm{cm}^{2}$ at $H_{\sim}=0.2 \mathrm{kOe}$ (curve 1) and $H_{\sim}=2.5 \mathrm{kOe}$ (curve 2).

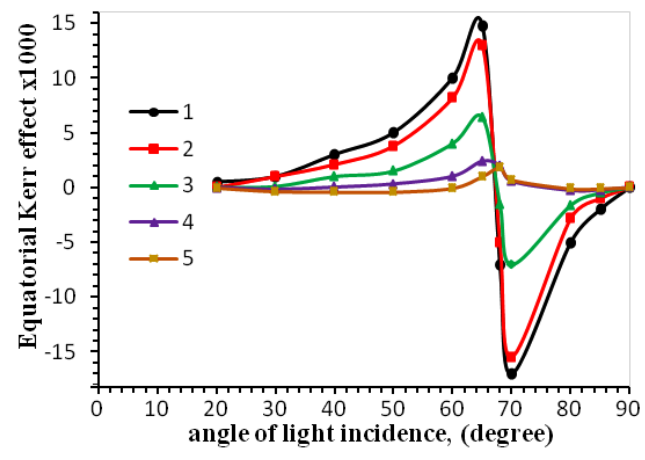

Fig. 3. Dependences of the EKE on the angle of light incidence $(\hbar \omega=2 \mathrm{eV})$ for the $(\mathrm{YBiCa})_{3}(\mathrm{FeGe})_{5} \mathrm{O}_{12}$ films before (curve 1) and after the process of implantation with various doses: $0.5 \times 10^{14}$ ion $/ \mathrm{cm}^{2}$ (curve 2), $1.5 \times 10^{14}$ ion $/ \mathrm{cm}^{2}$ (curve 3$), 2.0 \times 10^{14}$ ion $/ \mathrm{cm}^{2}$ (curve 4$)$ and $2.5 \times 10^{14}$ ion $/ \mathrm{cm}^{2}$ (curve 5).

Besides, the investigation of the dependences of the $\mathrm{EKE}$ on the angle in the region $2.6 \mathrm{eV}$ (Fig. 3) has shown that specific implantation dose $\left(1.5-2.5 \times 10^{14} \mathrm{ion} / \mathrm{cm}^{-2}\right.$ makes the change of a sign of the effect but it hardly has any influence on the position of the Brewster angle. This result has also been born out by the optical measurement which only proves the week relationship between the optical constants $n$ and $k$ and the implantation dose.

The research of magnetization processes of implanted films (Fig. 4) showed that transformation of the character of dependences in the region of light quantum energy $\hbar \omega=2.6 \mathrm{eV}$ might be explained by weakening antiferromagnetic ties between two iron sublattice and by turning over the magnetic moments of the iron ions which are responsible for the electronic transition in the external magnetic field.

From measurements of the EKE at two angles of light incidence of $\phi=70^{\circ}$ and $80^{\circ}$ and of the optical constants using the Avery method, we determined the spectral dependences of the components of the tensor of dielectric permittivity for the surface of the above-mentioned ferrite-garnet films before and after implantation process. 


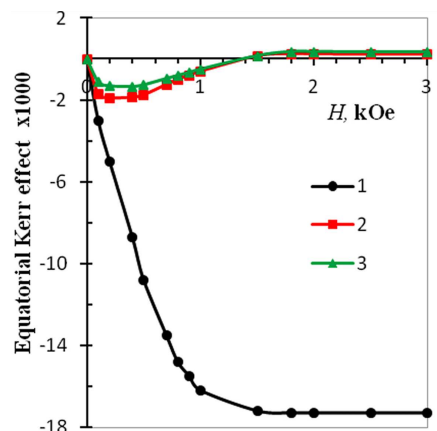

Fig. 4. Dependences of the EKE on the magnitude of the external alternating magnetic field $H_{\sim}(\hbar \omega=$ $2.6 \mathrm{eV})$ for the $(\mathrm{YBiCaSm})_{3}(\mathrm{FeGeSi})_{5} \mathrm{O}_{12}$ garnet films before (curve 1) and after the process of implantation with doses: $2.0 \times 10^{14}$ ion $/ \mathrm{cm}^{2}$ (curve 2) and $2.5 \times 10^{14}$ ion $/ \mathrm{cm}^{2}$ (curve 3 ).

These calculations let us evaluate the influence of the implantation on an electronic energy structure of the surface layer for the sample.

For examples, Figs. 5a,b and 6a,b give spectral dependences of the diagonal $\varepsilon_{1}$ and $\varepsilon_{2}$ and nondiagonal $\varepsilon_{1}^{\prime}$ and $\varepsilon_{2}^{\prime}$ components of the tensor of dielectric permittivity for the $(\mathrm{YBiCaSm})_{3}(\mathrm{FeGeSi})_{5} \mathrm{O}_{12}$ garnet films before and after the implantation with various doses.

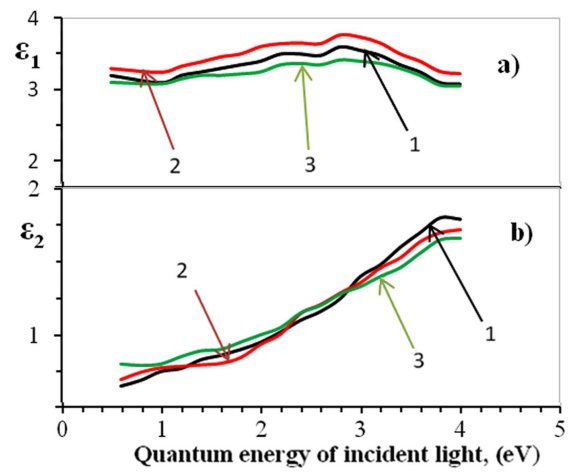

Fig. 5. Dependences of the diagonal $\varepsilon_{1}$ (a) and $\varepsilon_{2}$ (b) components of the tensor of dielectric permittivity on the quantum energy of incident light $\hbar \omega$ for the $(\mathrm{YBiCaSm})_{3}(\mathrm{FeGeSi})_{5} \mathrm{O}_{12}$ garnet films before (curve 1) and after the process of implantation with doses: $0.5 \times$ $10^{14}$ ion $/ \mathrm{cm}^{2}$ (curve 2), and $2.5 \times 10^{14}$ ion $/ \mathrm{cm}^{2}$ (curve 3 ).

It could be seen from the figures that for the films the spectral dependences of the diagonal components of the tensor of dielectric permittivity before and after implantation coincide (Fig. 5), whereas the character of the spectral dependences of the nondiagonal components of the tensor of dielectric permittivity essentially varies (Fig. 6).

On the spectral dependences of the imaginary part of the nondiagonal components of the tensor of dielectric permittivity, proportional density of electronic conditions, transformation of the character of dependences in the region of light quantum energy $\hbar \omega=2.6 \mathrm{eV}$ has distinctly been observed.

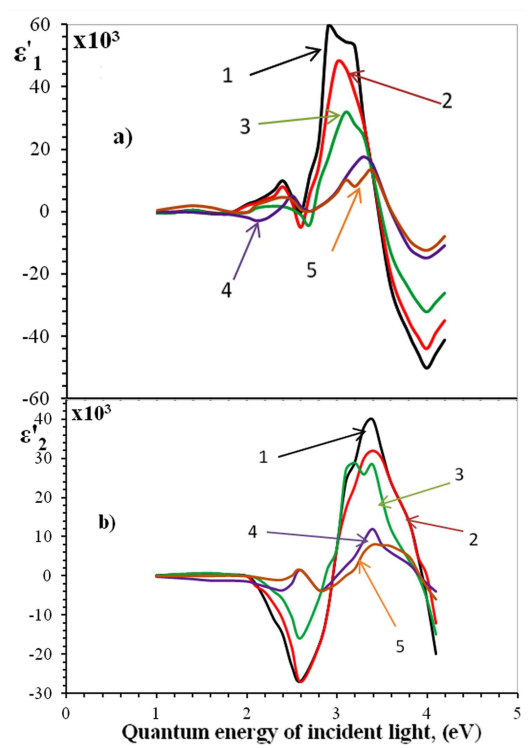

Fig. 6. Dependences of the nondiagonal $\varepsilon_{1}^{\prime}$ (a) and $\varepsilon_{2}^{\prime}$ (b) components of the tensor of dielectric permittivity on the quantum energy of incident light $\hbar \omega$ for the $(\mathrm{YBiCaSm})_{3}(\mathrm{FeGeSi})_{5} \mathrm{O}_{12}$ garnet films before (curve 1) and after the process of implantation with various doses: $0.5 \times 10^{14}($ curve 2$), 1.5 \times 10^{14}($ curve 3$), 2.0 \times 10^{14}$ (curve 4 ) and $2.5 \times 10^{14}$ ion $/ \mathrm{cm}^{2}$ (curve 5).

\section{Conclusion}

In the present work we have shown the efficiency of complex investigation of the ion implanted garnet surface region by magneto-optical and optical methods. From the analysis of the frequency dependences of the tensor of dielectric permittivity components received before and after implantation, we can conclude that implantation process destroys magnetic structure of garnet surface and essentially transforms electronic energy structure of implanted layer.

\section{References}

[1] L.V. Nikitin, L.G. Kalandadze, M.Z. Akhmedov, S.A. Nepijko, J. Magn. Magn. Mater. 148, 279 (1995).

[2] J.C. North, R. Wolfe, T.S. Nelson, J. Vac. Sci. Technol. 15, 1575 (1978).

[3] A.K. Zvezdin, V.A. Kotov, Magneto-Optics of Thin Films, Nauka, Moscow 1988, p. 81.

[4] G.S. Krinchik, Physics of Magnetic Phenomena, Moscow State University, Moscow 1985, p. 294.

[5] D. Avery, Proc. Phys. Soc. Lond. B 65, 426 (1952).

[6] L. Kalandadze, IEEE Trans. Magn. 44, 3293 (2008). 